\title{
Factores asociados con la picadura de alacrán en escolares: estudio transversal en dos comunidades rurales de Guerrero, México
}

\author{
Rufino Silva-Domínguez ${ }^{1}$, Sergio Paredes-Solís ${ }^{2 *}$, Antonio J. Cortés-Guzmán ${ }^{1}$, Miguel Flores-Moreno², \\ José G. Baldazo-Monsivaiz ${ }^{3}$, Neil Anderson ${ }^{2,4}$ y Anne Cockcroft ${ }^{4}$
}

${ }^{1}$ Secretaría de Salud de Guerrero, Chilpancingo, Guerrero, México; ${ }^{2}$ Centro de Investigación de Enfermedades Tropicales, Universidad Autónoma de Guerrero, Acapulco, Guerrero, México; ${ }^{3}$ Unidad Académica No. 13, Universidad Autónoma de Guerrero, Zihuatanejo, Guerrero, México; ${ }^{4}$ Departamento de Medicina Familiar, Universidad de McGill, Montreal, Canadá

\begin{abstract}
Resumen
Introducción: La intoxicación por picadura de alacrán es un problema de salud pública en las regiones de América Latina y América del Norte. El objetivo de este estudio fue identificar especies de alacrán de importancia médica, estimar la ocurrencia de accidentes por picadura de alacrán y factores asociados en la población de edad escolar. Métodos: Estudio transversal en dos comunidades rurales de Guerrero, México, en dos fases: el estudio entomológico para estimar índices escorpionológicos y la encuesta a escolares que incluyó las características de sus viviendas, la tenencia de aves, los antecedentes de picadura de alacrán y variables relevantes. Se estimó la razón de momios ajustada (RMa) como medida de asociación al accidente por picadura de alacrán en los escolares mediante análisis multivariado. Resultados: Las especies de importancia médica fueron Centruroides limpidus y Centruroides balsasensis. El reporte de accidente por picadura de alacrán en los últimos 6 meses en escolares fue del 12\% (171/1,437). Siete de cada diez escolares requirió de atención médica. Fueron identificados cuatro factores asociados con el reporte de accidente por picadura de alacrán: tener patos en la vivienda (razón de momios ajustada [RMa]: 1.98; intervalo de confianza [IC] 95\%: 1.2-4.29), falta de aseo en la vivienda (RMa: 1.84; IC 95\%: 1.02-4.16), ayudar en la labor del campo (RMa: 1.53; IC 95\%: 1.13-2.46) y viviendas con material del techo no permanente (RMa: 1.42; IC 95\%: 1.04-2.18). Conclusiones: Centruroides limpidus fue la especie de importancia médica más relevante. Fueron identificados dos factores asociados que son potencialmente modificables: mejorar el material del techo de la vivienda y asear la vivienda.
\end{abstract}

Palabras clave: Picadura de alacrán. Factores asociados. Escolares.

Factors associated with scorpion stings in schoolchildren: cross-sectional study from two rural communities of Guerrero, Mexico

\begin{abstract}
Introduction: Scorpion stings represent a public health problem in Latin America and North America. The aim of this study was to identify species of medical importance, as well as to estimate the frequency of scorpion sting and the associated risk
\end{abstract}

Disponible en internet: 18-03-2019 Bol Med Hosp Infant Mex. 2019;76:79-86 www.bmhim.com 
and protective factors in schoolchildren. Methods: Cross-sectional study in two rural communities in the Mexican state of Guerrero, in two phases: an entomological study that estimated the scorpion density and overcrowding index; a survey to document housing characteristics, poultry ownership, and history of scorpion sting in schoolchildren. Adjusted odds ratio (ORa) was used as a measure of association with scorpion sting in a multivariate analysis. Results: The species of medical importance were Centruroides limpidus and Centruroides balsasensis. Twelve percent $(171 / 1,437)$ of school children reported scorpion sting in the last six months, of whom seven out of ten required medical care. Four factors were associated with the report of scorpion sting: having ducks in the dwelling (ORa: 1.98; CI 95\%: 1.2-4.29), lack of cleanliness in the dwelling (ORa: 1.84; Cl 95\%: 1.02-4.16), farming as an occupation (ORa: 1.53; Cl 95\%: 1.13-2.46) and dwelling with uncovered infrastructure of the roof, those made of woodliron sheet or cardboard (ORa: 1.42; Cl 95\%: 1.04-2.18). Conclusions: The most relevant species was Centruroides limpidus. Two factors might be particularly relevant to prevention: improvement of household roofing material and attention to housekeeping.

Key words: Scorpion sting. Associated factors. Schoolchildren.

\section{Introducción}

La intoxicación por picadura de alacrán es un problema de salud pública en la región de América Latina y América del Norte ${ }^{1,2}$. En México, se registran alrededor de 300,000 casos y 40 defunciones anualmente ${ }^{3,4}$. La mortalidad en localidades con menos de 2,500 habitantes es casi doce veces mayor que la observada en las de más de 20,000 habitantes ${ }^{5}$. Los registros recientes de la Secretaría de Salud indican un aumento de su ocurrencia con tasas de 8,618 por 100,000 habitantes en 2012 y de 8,769 por 100,000 habitantes en 2013. El estado de Guerrero ocupó el primer lugar en intoxicaciones por picadura de alacrán durante el periodo de 2012 a 2014, el segundo lugar en el 2015 y el tercer lugar en $2016^{4}$.

Los alacranes se encuentran en casi todos los ambientes ecológicos. Se distribuyen principalmente en regiones tropicales y subtropicales, aunque se han adaptado a ambientes desérticos de clima caliente ${ }^{6}$. Hasta 2017, se tiene registro de 2,342 especies de alacranes en el mundo ${ }^{7}$, de las cuales solamente 50 especies son peligrosas para los humanos ${ }^{8}$. En México se conocen 289 especies, 19 de ellas de importancia médica ${ }^{9}$, que habitan principalmente en la vertiente del Océano Pacífico ${ }^{10}$.

Las especies tóxicas que existen en México pertenecen al género Centruroides de la familia Buthidae, y son responsables de cuadros clínicos graves o mortales $^{3,11}$. En Guerrero, durante mucho tiempo, la única especie de importancia médica conocida fue Centruroides limpidus ${ }^{12}$. Actualmente, se tiene registro de otras especies: Centruroides balsasensis, Centruroides tecomanus, Centruroides meisei, Centruroides villegasi ${ }^{13}$, Centruroides bonito ${ }^{14}$ y Centruroides poncei ${ }^{15}$. El veneno de las siete especies es altamente tóxico.
La mayor cantidad de accidentes por picadura de alacrán ocurre en poblaciones rurales, en el hogar y durante la noche ${ }^{8,16,17}$. Los hombres presentan mayor frecuencia de accidentes ${ }^{18}$ y la salud de los menores de 15 años es la más afectada ${ }^{17}$, con cuadros clínicos más graves en niños de 5 a 9 años de edad ${ }^{19,20}$. Las condiciones precarias de vivienda ${ }^{21}$ y caminar descalzo por la noche favorecen estos accidentes ${ }^{20}$. El uso de guantes en el trabajo de los campesinos adultos se ha encontrado como factor protector ${ }^{18} \mathrm{y}$, al parecer, no hay diferencia de los accidentes por picadura de alacrán según las condiciones socioeconómicas de las familias $^{22}$. El objetivo de este estudio fue identificar las especies de alacrán de importancia médica en dos comunidades de alta incidencia de intoxicación por picadura de alacrán, estimar la ocurrencia de estos accidentes en escolares y los factores asociados.

\section{Métodos}

Se realizó un estudio transversal en dos localidades rurales del estado de Guerrero, México: Zumpango del Río y Atliaca. Zumpango del Río es cabecera del municipio Eduardo Neri, se encuentra a $1,050 \mathrm{~m}$ sobre el nivel del mar en las coordenadas $17^{\circ} 39^{\prime} 11.70^{\prime \prime}$ latitud N y $99^{\circ} 31^{\prime} 99^{\prime \prime}$ longitud O. En la localidad viven aproximadamente 24,500 habitantes (51\% mujeres); todos hablan español. Zumpango cuenta con clima templado-tropical y lluvias de mayo a octubre. Su temperatura media es de $22^{\circ} \mathrm{C}$. La población tiene como actividades principales el comercio y la agricultura. Por su parte, la comunidad de Atliaca pertenece al municipio de Tixtla y está ubicada a 1,290 msnm en las coordenadas $17^{\circ} 39^{\prime} 10.57^{\prime \prime}$ latitud N y 99²2'23.64" longitud O. La habitan aproximadamente 7,200 personas (51\% mujeres). El $99 \%$ de la población es indígena. El 78\% de los habitantes habla español y náhuatl; el $15 \%$ habla solo español y el $7 \%$ 
Tabla 1. Fórmulas utilizadas para la estimación de índices escorpionológicos en las localidades de Zumpango del Río y Atliaca, Guerrero, México, 2013-2014

\begin{tabular}{|l|l|l|}
\hline Índice & Fórmula & Información que proporciona \\
\hline Índice de infestación & $=\frac{\text { Número de predios con alacranes }}{\text { Número de predios examinados }} \times 100$ & Grado de contacto persona-alacrán \\
\hline Índice de densidad & $=\frac{\text { Número de alacranes capturados }}{\text { Número de predios examinados }} \times 100$ & Distribución de los alacranes en la comunidad \\
\hline Índice de hacinamiento & $\frac{\text { Número de alacranes capturados }}{\text { Número de predios positivo de alacranes }} \times 100$ & Densidad relativa de alacranes en predios positivos \\
\hline
\end{tabular}

Fuente: Norma Oficial Mexicana NOM-033-SSA2-2011. Para la vigilancia, prevención y control de la intoxicación por picadura de alacrán. Publicada en el Diario Oficial de la Federación de México el 22 de noviembre de 2011.

habla solo náhuatl. El clima es cálido y seco, con una temperatura que oscila entre los 25 y los $37^{\circ} \mathrm{C}$. La agricultura es la principal ocupación de los pobladores.

El estudio comprendió dos fases: la primera, de julio de 2013 a junio de 2014, para identificar especies de alacranes; la segunda, de abril a junio de 2016, para obtener información de los escolares relacionada con la ocurrencia de accidente por picadura de alacrán y los factores asociados.

\section{Estudio entomológico}

La recolecta de alacranes se realizó en diez colonias periféricas de Zumpango del Río y en seis de Atliaca. Se aplicaron métodos de captura directos diurnos, mediante la detección activa de alacranes, buscando principalmente en techos, paredes, cortezas de postes de cerca, bajo láminas de cartón y rocas y en tejas apiladas. Los datos se registraron en el formato ALER-1 que utiliza la Secretaría de Salud ${ }^{23}$. Para la identificación de la especie, se siguieron las claves publicadas por Baldazo, et al. ${ }^{13}$ y Ponce, et al. ${ }^{9}$ Con los especímenes recolectados se obtuvieron los índices de infestación, densidad y hacinamiento ${ }^{24}$, según las formulas descritas en la tabla 1.

\section{Encuesta a escolares}

El tamaño de la muestra fue calculado con intervalo de confianza del 95\% (IC 95\%), poder del estudio del $80 \%$, tasa esperada del evento del $10 \%$ y razón de momios (RM) mínima a detectar de 2.0. El resultado del número mínimo de observaciones fue 948 . Por conveniencia logística, se incluyeron escolares de todas las escuelas de educación primaria de las localidades, lo cual garantizó cubrir el tamaño mínimo de muestra estimado.

Para estimar la ocurrencia de accidente por picadura de alacrán y los factores asociados, se diseñó un instrumento de medición que consistió en un cuestionario con 33 preguntas, el cual fue validado mediante una ronda de expertos ${ }^{25}$. El grupo de diseño se conformó por un entomólogo médico, un maestro en ciencias (ambos con experiencia en trabajos de alacranismo) y dos epidemiólogos, uno de la Secretaría de Salud de Guerrero y otro de la Universidad Autónoma de Guerrero.

El cuestionario se aplicó un día ordinario de asistencia a clases a escolares de cuarto a sexto grado de educación primaria de las escuelas existentes en las localidades, tres en Atliaca y ocho en Zumpango del Río. Un facilitador leyó las preguntas y los escolares seleccionaron y marcaron sus respuestas. Se recolectó información sobre la edad, sexo y grado de los escolares, condiciones y materiales de su vivienda, tenencia de aves, conocimiento de los alacranes y su percepción de peligrosidad y consecuencias del contacto con ellos y antecedentes de picaduras por alacrán a miembros de su familia $o$ al escolar. Con respecto a la picadura experimentada por el escolar, se preguntó sobre la fecha de la última vez que ocurrió, parte del cuerpo afectada, color del alacrán, sitio donde estaba el alacrán, lugar donde se encontraba el escolar, síntomas que presentó, lugar donde lo atendieron, tratamiento utilizado, costo y si requirió hospitalización. Además, se preguntó sobre su percepción de acciones preventivas contra la picadura de alacrán y el antecedente de muertes en su familia por intoxicación de picadura de alacrán. Para el análisis, se consideró como variable resultado 
Tabla 2. Principales resultados del estudio entomológico en las localidades de Zumpango del Río y Atliaca, Guerrero, México, 2013-2014

\begin{tabular}{|l|c|c|}
\hline Indicador entomológico & $\begin{array}{c}\text { Zumpango del } \\
\text { Río }\end{array}$ & Atliaca \\
\hline Predios revisados & 70 & 60 \\
\hline $\begin{array}{l}\text { Predios positivos a } \\
\text { alacranes }\end{array}$ & 40 & 40 \\
\hline Especímenes capturados & $\begin{array}{c}445 \\
\text { alacranes }\end{array}$ & $\begin{array}{c}465 \\
\text { alacranes }\end{array}$ \\
\hline Índice de infestación & 57 & 67 \\
\hline Índice de densidad & 636 & 623 \\
\hline Índice de hacinamiento & 1,113 & 935 \\
\hline Especímenes identificados & 350 & 374 \\
\hline $\begin{array}{l}\text { Principales especies de } \\
\text { alacrán identificadas }\end{array}$ & & alacranes \\
\hline Centruroides limpidus & 344 & 364 \\
\hline Mesomexovis variegatus & 1 & 2 \\
\hline Centruroides balsasensis & 3 & - \\
\hline Mesomexovis atenango & 1 & - \\
\hline Hoffmannihadrurus gertschi & 1 & - \\
\hline Vaejovis curvidigitus & - & - \\
\hline 5 acran & & \\
\hline
\end{tabular}

ag5 especímenes no fueron identificados por ser ejemplares juveniles o por estar deteriorados.

b91 especímenes no fueron identificados por ser ejemplares juveniles o por estar deteriorados.

la ocurrencia del reporte de accidentes por picadura de alacrán en los últimos 6 meses.

El análisis se llevó a cabo con el paquete estadístico CIETmap ${ }^{26}$. Con el proceso de Mantel-Haenszel se estimó la RM y el IC 95\% de las variables incluidas en el análisis bivariado y multivariado ${ }^{27}$. Los IC 95\% fueron ajustados por conglomerado utilizando el método propuesto por Lamothe ${ }^{28}$. El análisis multivariado se utilizó para estimar asociaciones con efecto independiente; se inició con el modelo saturado, que incluyó las variables que alcanzaron significación estadística, eliminando uno por uno los factores que no mantuvieron confianza estadística $(p<0.05)$ hasta quedar con el modelo final.

La información recolectada fue confidencial, anónima y las autoridades escolares y padres o tutores autorizaron la aplicación de la encuesta. A los escolares les fue explicado el objetivo del estudio y ellos decidieron contestar o no el cuestionario. El protocolo fue registrado y autorizado en el Comité Local de Investigación de la
Tabla 3. Características de la vivienda de los escolares de Atliaca y Zumpango del Río, Guerrero, México, 2016

\begin{tabular}{|l|c|c|}
\hline Factor & $\mathbf{N}=\mathbf{1 , 4 3 7}$ & $\%$ \\
\hline $\begin{array}{l}\text { Paredes de la vivienda } \\
\text { Cemento }\end{array}$ & 721 & 50 \\
\hline $\begin{array}{l}\text { Ladrillo } \\
\text { Adobe } \\
\text { Madera } \\
\text { Lámina }\end{array}$ & 430 & 30 \\
\hline $\begin{array}{l}\text { Piso de la vivienda } \\
\text { Cemento }\end{array}$ & 177 & 5 \\
\hline $\begin{array}{l}\text { Loseta } \\
\text { Tierra }\end{array}$ & 33 & 2 \\
\hline $\begin{array}{l}\text { Techo de la vivienda } \\
\text { Cemento }\end{array}$ & 463 & 58 \\
\hline $\begin{array}{l}\text { Teja } \\
\text { Lámina/madera/cartón }\end{array}$ & 143 & 10 \\
\hline Usa leña para cocinar & 813 & 32 \\
\hline Guarda leña dentro de la vivienda & 92 & 44 \\
\hline Usa cielo arriba de la cama & 608 & 24 \\
\hline $\begin{array}{l}\text { Guarda material de construcción dentro } \\
\text { de la vivienda }\end{array}$ & 642 & 43 \\
\hline
\end{tabular}

Facultad de Ciencias Químico Biológicas y del Centro de Investigación de Enfermedades Tropicales de la Universidad Autónoma de Guerrero.

\section{Resultados}

La principal especie de importancia médica identificada en ambos municipios fue Centruroides limpidus. El $98 \%$ de los especímenes identificados en Zumpango del Río (344/350) y en Atliaca (364/374) correspondieron a esta especie. Los resultados más relevantes del estudio entomológico se muestran en la tabla 2.

Se encuestaron 1,437 escolares de cuarto a sexto grado de educación primaria, el 24\% $(348 / 1,437)$ de las escuelas de Atliaca y el $76 \%(1,089 / 1,437)$ de Zumpango del Río. El 49\% (699/1,437) de los escolares fue del sexo masculino; el promedio de edad fue 10.6 años (rango 9 a 13). La tabla 3 muestra algunas características de la vivienda de los escolares.

El $97 \%(1,394 / 1,437)$ de los escolares conoce los alacranes y el $94 \%(1,351 / 1,437)$ los considera peligrosos. El $85 \%(1,221 / 1,429)$ de los encuestados sabe que debe acudir al médico cuando se produce una picadura por alacrán. El 25\% $(359 / 1,437)$ conoce que debe sacudir su ropa, el 16\% $(227 / 1,437)$ que debe revisar sus zapatos antes de usarlos y el $40 \%(577 / 1,435)$ usa cielo (protección) sobre sus camas. 
Tabla 4. Análisis bivariado de factores asociados a reporte de picadura de alacrán en los últimos 6 meses en escolares de Atliaca y Zumpango del Río, Guerrero, México, 2016

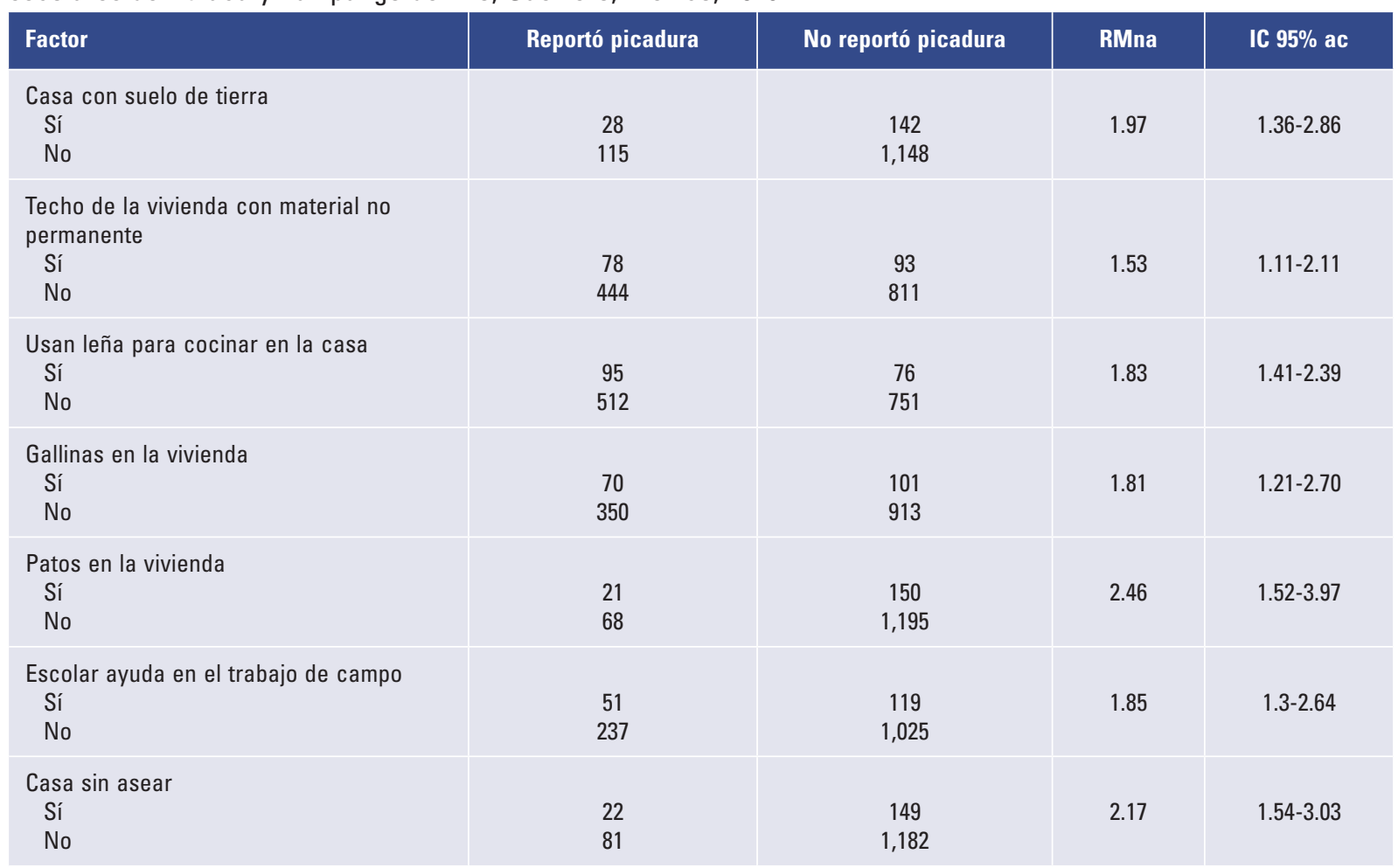

IC95\% ac: intervalo de confianza del 95\% ajustado por conglomerado; RMna: razón de momios no ajustada.

El 36\% $(522 / 1,437)$ de los escolares reportó al menos un accidente por picadura por alacrán en su vida. El reporte de accidente por picadura de alacrán en los últimos 6 meses fue del 12\% (171/1,435): el 13\% $(91 / 697)$ en niños y el $11 \%(80 / 737)$ en niñas $(p=0.19)$. El 5\% $(68 / 1,436)$ de los escolares reportó que algún familiar murió por picadura de alacrán. El 45\% (233/522) de los encuestados con al menos un accidente por picadura de alacrán mencionó que el alacrán fue de color amarillo, mientras que el $54 \%$ (284/522) sufrió la picadura en la mano/brazo y el $36 \%$ (190/522) en la pierna/pie. En el momento de ocurrir la picadura, el $65 \%(340 / 522)$ se encontraba dentro de su casa, el $15 \%(79 / 522)$ en el patio, el $2 \%(9 / 522)$ en la escuela, el $6 \%(32 / 522)$ en el campo y el $12 \%(62 / 522)$ en un lugar no especificado. El 68\% (355/522) fue atendido en un hospital o centro de salud, mientras que el $30 \%$ $(156 / 522)$ en su domicilio con remedios caseros y el $1.4 \%(6 / 522)$ por curanderos. El $61 \%(317 / 522)$ de los casos se trataron con faboterápico y 249 escolares (48\%) fueron hospitalizados.

Por otro lado, el $4 \%(23 / 522)$ de los escolares con accidente por picadura de alacrán informó que no presentó síntomas. En los escolares con sintomatología, el 33\% (165/499) refirió dolor leve, el 36\% (181/499) padeció dolor intenso en el sitio de la picadura y el $31 \%$ (153/499) presentó uno o varios de los síntomas siguientes: vómito, pérdida de la percepción de colores, sensación de cuerpo extraño en la faringe y desmayo.

En cuanto a los costos, el 78\% (409/522) de escolares con accidente por picadura de alacrán desconocía el monto de la atención y el 4\% (21/522) refirió que la atención fue gratuita. Para 74 casos (14\%), el costo tuvo un rango de 10 a 1,000 pesos mexicanos.

El análisis bivariado identificó siete variables asociadas con el reporte de picadura de alacrán en los últimos 6 meses. Dos factores están relacionados a las condiciones de la vivienda, material del suelo y techo; otros dos, a la tenencia de aves en la vivienda, gallinas o patos, y tres a hábitos de la familia: cocinar con leña, que los niños ayuden en el trabajo de campo y la casa no aseada. La tabla 4 muestra los factores asociados identificados en el análisis bivariado con la estimación de la RM y del IC 95\% ajustado por conglomerado (IC 95\% ac).

En el modelo final de análisis multivariado, cuatro factores se mantuvieron con efecto independiente 
Tabla 5. Modelo final del análisis multivariado de factores asociados a reporte de picadura de alacrán en los últimos 6 meses en escolares de Atliaca y Zumpango del Río, Guerrero, México, 2016

\begin{tabular}{|l|c|c|c|c|c|}
\hline Factor & RMna & RMa & IC 95\%ac & X $^{2 \text { het }}$ & p \\
\hline Patos en la vivienda & 2.15 & 1.98 & $1.20-4.29$ & 10.37 & 0.99 \\
\hline Casa sin asear & 1.86 & 1.84 & $1.02-4.16$ & 8.03 & 0.92 \\
\hline Escolar ayuda en el trabajo de campo & 1.71 & 1.53 & $1.13-2.46$ & 7.86 & 0.54 \\
\hline Techo de la vivienda con material no permanente & 1.46 & 1.42 & $1.04-2.18$ & 6.09 & 0.83 \\
\hline
\end{tabular}

IC: intervalo de confianza del 95\% de la razón de momios ajustada por conglomerado; RMa: razón de momios ajustada; RMna: razón de momios no ajustada; p: valor de p de la chi cuadrada de heterogeneidad; $X^{2 \text { het: }}$ Chi cuadrada de heterogeneidad.

sobre el reporte de accidente por picadura de alacrán en los últimos 6 meses. La mayor fuerza de asociación se presentó con el factor tenencia de patos en la vivienda (razón de momios ajustada (RMa): 1.98; IC 95\% ac: 1.20-4.29), después continuaron los factores aseo de la vivienda, que el escolar ayude en el trabajo de campo y el material no permanente del techo de la casa. La tabla 5 presenta los detalles de la estimación de RMa e IC 95\% ac, del análisis multivariado.

\section{Discusión}

La principal especie de alacrán de importancia médica hallada fue Centruroides limpidus. El reporte de accidente por picadura de alacrán en los últimos 6 meses fue del $12 \%$ en los escolares, y los factores asociados fueron tenencia de patos, casa sin asear, ayudar en las labores del campo y vivir en una casa con techo de material no permanente.

En Guerrero, la especie de alacrán de importancia médica que más predomina es Centruroides limpi$d u s^{11}$, aunque existen otras especies en menor proporción. Casi todos los alacranes recolectados en el estudio entomológico correspondieron a Centruroide limpidus y solamente se identificaron tres especímenes de Centruroide balsasensis en Zumpango del Río. Por este hallazgo, se considera alta la probabilidad de que los accidentes por picadura de alacrán reportados por los escolares impliquen a Centruroides limpidus, un alacrán de alta toxicidad ${ }^{12}$.

Los índices escorpionológicos en ambas localidades fueron similares. El índice de infestación señala la convivencia cercana del alacrán con los humanos y el índice de densidad sugiere distribución amplia de los alacranes en la comunidad. El índice de hacinamiento revela una alta aglomeración de alacranes por domicilio. Los resultados de los índices sugieren que las localidades tienen condiciones apropiadas como hábitat para los alacranes.
Actualmente no existen parámetros que clasifiquen el nivel de riesgo a accidente por picadura de alacrán de acuerdo con los índices escorpionológicos. En particular, se seleccionaron estas localidades con base en los registros de intoxicación por picadura de alacrán de la Secretaría de Salud de Guerrero. Los hallazgos de los índices escorpionológicos confirman que se eligieron comunidades con alto grado de exposición a este tipo de accidentes. La recolecta de alacranes fue realizada durante el día, ya que no existían las condiciones de seguridad para hacerlo por las noches. En este sentido, cabe destacar que los índices están subestimados, ya que los alacranes son de hábitos nocturnos.

El reporte de los escolares entrevistados sobre el color del alacrán con el cual tuvieron el accidente (amarillo), permite determinar que corresponderían a las especies Centruroides limpidus y Centruroides balsasensis, que son los únicos con estas características; las demás especies son de color marrón. El $80 \%$ de los accidentes ocurrieron dentro y alrededor del domicilio, lo cual se explicaría por el alto índice de hacinamiento de alacranes. Una de cada tres picaduras fue en el pie de los escolares; se ha reportado que andar descalzo favorece estos accidentes ${ }^{17}$, pero en el presente estudio no se preguntó acerca del uso de calzado. Siete de cada diez niños con picadura reportó que requirió ser atendido en un hospital o centro de salud, lo que indica la gravedad de la intoxicación, otra característica de los accidentes con Centruroides limpidus ${ }^{6}$.

Dos de los cuatro factores asociados con la picadura por alacrán del modelo final de análisis multivariado representan condiciones de la vivienda: la posesión de patos y el aseo de la vivienda. Las otras dos condiciones socioeconómicas de la familia son el material no permanente del techo de la casa y que los escolares tengan que ayudar con el trabajo del campo. Se considera que las familias con una vivienda no permanente (lámina, madera o cartón) y en las que los 
escolares se incorporen al trabajo del campo, representan a las poblaciones más vulnerables desde el punto de vista económico; este hallazgo resulta diferente al reportado por Almeida et al., en Brasil ${ }^{22}$. Se conoce que las condiciones precarias de las viviendas favorecen los accidentes por picadura de alacrán ${ }^{17}$.

Por ser un diseño transversal, este estudio tiene la limitante de la temporalidad entre exposición y efecto. Razonablemente, se esperaría que los factores asociados identificados (el aseo de la vivienda, el material del techo y la necesidad de ayuda en el trabajo del campo) precedan a la picadura. Sin embargo, no es el caso para la asociación del accidente con la tenencia de patos. Existe la versión, no documentada, de que los patos se alimentan de alacranes y que, por tener un pico largo y de material impenetrable por el aguijón del alacrán, son poco vulnerables a la picadura. Si esta versión fuese correcta, la asociación explicaría la presencia de los patos como respuesta de las personas para reducir la densidad de alacranes en la vivienda. Otra posibilidad que se propone es que la presencia de estas aves y su alojamiento, junto con el material necesario para ello (principalmente maderas, cartón y paja), crean condiciones favorables para el hábitat del alacrán. Otras investigaciones deben explorar estas hipótesis.

Desde el punto de vista epidemiológico, las condiciones de las viviendas son importantes para que ocurran accidentes de picadura de alacrán. Los encuestados reportaron que las paredes de sus casas son de ladrillo, madera, adobe y lámina, y los techos de material no permanente, como la lámina y la madera, que son sitios preferenciales como refugio de escorpiones.

Aunque el estudio no tuvo la intención de medir detalles de los costos de la atención médica, la mayoría de los escolares desconocían la cantidad pagada por el tratamiento y atención de la picadura del alacrán. Los resultados de la frecuencia con la que se requiere utilizar los servicios de salud, el costo de los tratamientos, los días de hospitalización e incapacidad de los afectados en los casos graves demandan investigaciones puntuales sobre la estimación de los costos en que incurren las familias y los servicios de salud.

Mejorar las condiciones de la vivienda y evitar que los niños laboren en el campo representan un costo para las familias. Sin embargo, el presentar los resultados del costo que conlleva la atención de una persona intoxicada por picadura de alacrán proporciona, al menos, un marco de comparación entre costo y beneficio de su decisión. Además, este dato también serviría para proteger al resto de los integrantes de la familia. Los mismos resultados sirven a las autoridades gubernamentales para reorientar el gasto público en los municipios donde las intoxicaciones por picadura de alacrán son un problema importante.

\section{Responsabilidades éticas}

Protección de personas y animales. Los autores declaran que para esta investigación no se han realizado experimentos en seres humanos ni en animales.

Confidencialidad de los datos. Los autores declaran que han seguido los protocolos de su centro de trabajo sobre la publicación de datos de pacientes.

Derecho a la privacidad y consentimiento informado. Los autores declaran que en este artículo no aparecen datos de pacientes.

\section{Fuente de financiamiento}

\section{Ninguna.}

\section{Agradecimientos}

A los padres de familia y escolares que colaboraron con nosotros y compartieron sus experiencias. A las autoridades civiles y educativas de Zumpango del Río y Atliaca, reconocemos su compromiso con la salud escolar. Agradecemos a Lucero Andraca Ponce, Abigail Silva Bedolla y Félix Calzada García, de la Licenciatura de Biología de la Facultad de Ciencias Químico Biológicas de la Universidad Autónoma de Guerrero, por su colaboración en el trabajo de campo de este estudio.

\section{Bibliografía}

1. Borges A, Miranda JR, Pascale JM. Scorpionism in Central America, with special reference to the case of Panama. J Venom Anim Toxins incl Trop Dis. 2012;18:130-43

2. Isbister GK, Bawaskar HS. Scorpion envenomation. N Engl J Med. 2014; 371:457-63.

3. Villa-Manzano AI, Vázquez-Solís MG, Zamora-López XX, Arias-Corona F, Palomera-Ávila FM, Pulido-Galaviz C, et al. Alacranismo severo causante de parálisis flácida aguda. Reporte de caso. Rev Med Inst Mex Seguro Soc. 2016:54:265-8.

4. Secretaría de Salud. Boletín Epidemiológico. Sistema Nacional de Vigilancia Epidemiológica. Sistema Único de Información. 2017. Disponible en: https://www. gob.mx/salud/acciones-y-programas/direccion-general-de-epidemiologia-boletin-epidemiologico.

5. Celis A, Gaxiola-Robles R, Sevilla-Godínez E, Orozco Valerio MJ, Armas J. Tendencia de la mortalidad por picaduras de alacrán en México, 1979-2003. Rev Panam Salud Publica. 2007;21:373-80.

6. Possani LD. El alacrán y su piquete. Dirección General de Divulgación de la Ciencia, UNAM; 2005.

7. Rein JO. The scorpion files. Trondheim, Noruega University of Science and Technology; 2017. Disponible en: http://ntnu.no./ub/scorpion-files/.

8. Silva MSV, Silva Cláudio GL, Silva Neto B, Grangeiro Júnior CRP Lopes VHG, Teixeira Júnior AG, et al. Clinical and epidemiological aspects of scorpionism in the world: a systematic review. Wilderness Environ Med. 2016;27:504-18.

9. Ponce-Saavedra J, Francke BOF, Quijano-Ravell AF, Cortés Santillán R. Alacranes (arachnida: escorpiones) de importancia para la salud pública en México. Folia Entomol Mex. 2016;2:45-70.

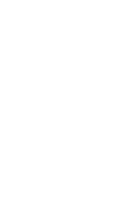

(1) 
10. Santibañez-López CE, Francke OF, Ureta C, Possani LD. Scorpions from Mexico: from species diversity to venom complexity. Toxins (Basel). 2015;8:pii: E2.

11. Quijano-Ravell AF, Ponce-Saavedra J, Francke OF, Villaseñor-Ramos MA. Nuevos registros y distribución actualizada de Centruroides tecomanus Hoffmann, 1932 (Scorpiones: Buthidae). Ciencia Nicolaita. 2010;52: 179-89.

12. Villegas A, Andersson N, Martinez E. Alacranismo en Guerrero: un estudio epidemiológico en 20 comunidades. Salud Pública Mex. 1988;30: 234-9.

13. Baldazo-Monsivaiz JG, Ponce-Saavedra J, Flores-Moreno M. Alacranes (arachnida: scorpionida) de importancia médica del estado de Guerrero, México. Dugesiana. 2012;19:143-50

14. Quijano-Ravell AF, Teruel R, Ponce-Saavedra J. A new Centruroides Marx, 1890 (Scorpiones: Buthidae), from Southern Guerrero State, Mexico. Rev Iber Aracnol. 2016;8:25-34.

15. Teruel R, Kovarik F, Baldazo-Monsivaiz JG, Hoferek D. A new species of Centruroides of the "nigrovariatus" group (Scorpiones: Buthidae) from southern Mexico. Revista Iber Aracnol. 2015;26:3-14.

16. Ebrahimi V, Handami E, Moemenbellah-Fard MD, Ezzatzadegan-Jahromi E. Predictive determinats of scorpion sting in a tropical zone of south Iran: use of mixed seasonal autoregressive moving average model. J Venom Anim Toxins Incl Trop Dis. 2017;23:39.

17. Celis A, Gaxiola-Robles R, Sevilla-Godínez E, Orozco Valerio MJ Armas J. Tendencia de la mortalidad por picaduras de alacrán en México, 1979-2003. Rev Panam Salud Pública. 2007;21:373-80.

18. Villegas-Arrizón A, Garzón-Mayo R, Flores-Moreno M, Andersson N. E uso de guantes como factor protector contra picaduras de alacrán durante la pizca de maíz en el estado de Guerrero, México. Salud Pública Mex. 2009;51:126-33.
19. Guerra CM, Carvalho LF, Colosimo EA, Freire HB. Analysis of variables related to fatal outcomes of scorpion envenomation in children and adolescents in the state of Minas Gerais, Brazil, from 2001 to 2005. J Pediatr (Rio J). 2008;84:509-15.

20. Bahloul M, Chabchoub I, Chaari A, Chtara K, Kallel H, Dammak H, et al. Scorpion envenomation among children: clinical manifestations and outcome (analysis of 685 cases). Am J Trop Med Hyg. 2010;83:1084-92.

21. Consejo Nacional de Evaluación de la Política de Desarrollo Social. Disponible en: http://www.coneval.org.mx/coordinacion/entidades/Guerrero/Paginas/carencias-sociales20102015.aspx.

22. Almeida TS, Fook SM, França FO, Monteiro TM, Silva EL, Gomes LC et al. Spatial distribution of scorpions according to the socioeconomic conditions in Campina Grande, State of Paraíba, Brazil. Rev Soc Bras Med Trop. 2016:49:477-85.

23. Thirión Icaza J, Losoya Solís A. Entomología con énfasis en control de vectores. Manual de procedimientos para el estudio entomológico de alacranes y su registro. México: Secretaría de Salud; 1997.

24. Norma Oficial Mexicana NOM-033-SSA2-2011. Para la vigilancia, prevención y control de la intoxicación por picadura de alacrán. Diario Oficial de la Federación; 2011.

25. Escobar-Pérez J, Cuervo-Martínez A. Validez de contenido y juicio de expertos: una aproximación a su utilización. Avances en Medición. 2008;6:27-36.

26. Andersson N, Mitchell S. CIETmap: Free GIS and epidemiology software from the CIETgroup, helping to build the community voice into planning. Montreal: World Congress of Epidemiology; 2002.

27. Mantel N, Haenszel W. Statistical aspects of the analysis of data from retrospective studies of disease. J Natl Cancer Inst. 1959:22:719-48.

28. Lamothe $\mathrm{G}$. Adjusting the Mantel Haenszel test statistic and odds ratio for cluster sampling. BMC Health Serv Res. 2011;11:S2-15. 